\title{
Management of intra uterine growth restriction
}

\author{
JH du Plessis', HL Chauke* \\ Registrar ${ }^{1}$ and Consultant*, Department of Obstetrics and Gynaecology, University of Pretoria, Pretoria, South Africa
}

\begin{abstract}
Introduction
Intra-uterine growth restriction (IUGR) remains a complex management problem in modern obstetric practise. It is a major cause of perinatal morbidity and mortality in South Africa as well as in the developed world and is a cause of great concern for the patient, her family and doctor.

Insufficient growth that result from intrinsic fetal factors such as aneuploidy, congenital malformations and congenital infection carry a guarded prognosis that often cannot be improved by a therapeutic intervention. In comparison, IUGR related to inadequate substrates for fetal metabolism and decreased oxygen availability has a better prognosis. ${ }^{1}$

The challenge to the obstetrician is to identify when inadequate growth occurs abdto determine the cause and severity. This will enable fair and proper counselling of the parents concerning possible outcome and prognosis. Decisions have to be taken regarding fetal monitoring and growth evaluation and timely delivery, this after consultation with the paediatrician or neonatologist.
\end{abstract}

\section{INITIAL DIAGNOSTIC EVALUATION}

The various causes, management options and ultimate prognosis of fetuses with IUGR differ widely and it is important to examine each case individually to look for maternal, placental or fetal disorders. This is achieved by obtaining a complete history, doing a thorough physical examination, ultrasound and other special investigations.

\section{Complete History}

In addition to the normal aspects of the history, detailed attention should be given to the following:

- fetal growth restriction in previous pregnancies, because of the risk for recurrence in subsequent pregnancies

- maternal or family history of thrombosis as this can be associated with congenital and acquired trombophilia disorders.

- social habits that can play a role namely smoking, use of alcohol and of recreational drugs.

\section{Physical Examination}

In addition to the standard clinical assessment the following aspects are important:

- Clinical suspicion of viral infections like Cytomegalovirus, Rubella, Varicella Zoster and Herpes Simplex virus must be further investigated by maternal serum testing followed, if positive, by amniotic fluid testing for DNA.

- TORCH screening should not be done routinely unless non-specific ultrasound markers of abnormality including echogenicity and calcification of the brain and liver as well as hydrops are present on sonar assessment.

Screening for inadequate growth in a low risk population is commonly performed using the symphysis-fundus height (SFH) measurement. This however has a varying sensitivity of 27-85\%. Up to 50\% of small for gestational age fetuses are not suspected antenataly. ${ }^{2}$ In women with risk factors for fetal growth restriction, ultrasound assessment of fetal growth must be done in addition to serial SFH.

\section{Imaging}

Accurate dating of the pregnancy forms part of the cornerstone of the diagnosis of IUGR. Ideally duration of gestation must be confirmed by crown rump length measurement $(\mathrm{CRL})$ during the first trimester or by second trimester biometry.

Estimation of fetal weight (EFW) is a critical element in diagnosis and monitoring. The EFW on ultrasound will be within $15 \%$ of the true weight in $95 \%$ of cases. Serial measurements rather than single estimates of the abdominal circumference and EFW are recommended. ${ }^{3}$ This can be performed every 2 weeks in order to reduce false positive values due to ultrasound errors.

The Royal College of Obstetricians and Gynaecologists (RCOG) recommends plotting of values on customized growth charts. ${ }^{4}$ In compiling these customised growth curves physiological factors known to affect fetal size are incorporated, such as gender, maternal weight, height and ethnicity. It appears to give better results than general population curves at identifying those small fetuses at risk of increased perinatal complications. ${ }^{5}$

Detailed anatomical survey of the fetus is essential due to the association of major fetal anomalies with IUGR. Growth 
restriction is found among $20-60 \%$ of malformed infants. In comparison, 10\% of IUGR is accompanied by congenital abnormalities. ${ }^{6}$

The anomalies associated with growth restriction include: omphalocoele, diaphragmatic hernia, skeletal dysplasia, congenital heart defects, microcephaly (rare), polyhydramnios (associated with trisomy 18). Once intrinsic fetal abnormalities have been excluded, placental insufficiency usually remains the presumed aetiological diagnosis.

\section{Laboratory Evaluation/ Special Investigations}

Fetal karyotyping is indicated in the following scenarios:

- early $(<32$ weeks) detection of IUGR

- severe IUGR ( $<3$ rd percentile)

- IUGR accompanied by polyhydramnios (to exclude Trisomy 18)

- structural abnormalities found during ultrasound examination. In 10-14\% of structural abnormal growth restricted fetuses the karyotyping will be abnormal. However, only $2 \%$ of structurally normal growth fetuses have chromosomal abnormalities.

Trisomy 18, trisomy 13 and triploidy are associated with early onset IUGR. Some of the ultrasound markers suggestive of aneuploidy include echogenic bowel, nuchal fold thickening and abnormal hand positioning.

After initial assessment constitutionally small fetuses should be differentiated from truly growth restricted fetuses. These constitutionally small fetuses are anatomically normal with appropriate amniotic fluid volumes and growth rates. The outcome for this group is good. It is important to recognize ethnic and geographic differences in growth potential. This will prevent unnecessary interventions in these patients.

\section{SUBSEQUENT OBSTETRICAL MANAGEMENT ANTENATAL SURVEILLANCE}

The purpose of antenatal surveillance is to identify fetuses at highest risk of in-utero demise, who may benefit from intervention by preterm delivery.

\section{ULTRASOUND}

This is core to decision making. Growth as well as arterial and venous blood flow in the fetus can be assessed.

The frequency of serial examination must be based on severity of findings:

- monitor fetal well-being - 1-7 times per week

- fetal growth - 2-4 week

Growth is plotted on customized growth curves (where available) to detect when growth is below the 10th percentile, and in order to follow growth progress.

Doppler velocimetry is the primary surveillance tool in pregnancy with suspected IUGR.

\section{UMBILICAL ARTERY (UA) DOPPLER}

UA Doppler studies are used to stratify SGA infants into low and high risk categories. They play an important role in the initial confirmation of placental insufficiency and in ongoing surveillance and management.

It has been demonstrated that detection of abnormalities in Doppler waveforms can predict those small for gestational age babies at highest risk of prenatal mortality, with the risk of death correlating with severity of Doppler waveform deviation: odds ratio $=4.0$ with absent end diastolic flow $(\mathrm{AEDF})$ and reversed end diastolic flow (REDV), odds ratio = 10.6 for perinatal mortality. ${ }^{7}$ It also influences the risk for cerebral haemorrhage, anaemia and hypoglycaemia in the neonate with increased neonatal morbidity.

Numerous randomized controlled trials showed that using Doppler velocimetry perinatal death and unnecessary induction of labour are significantly reduced. ${ }^{1}$ Meta-analysis found that clinical action guided by Doppler ultrasound reduces the odds of prenatal death by $38 \%$ in high risk pregnancies. There were however no advantage in using this tool for screening in a low risk population. ${ }^{8}$

The SGA fetus with normal UA Doppler findings is unlikely to have major morbidity (normal and slightly increased umbilical Doppler flow) and it is safe to prolong and also reduce surveillance. A large prospective trial is required to determine the appropriate amount of surveillance for SGA infant with normal UA Doppler.

\section{MIDDLE CEREBRAL ARTERY (MCA) DOPPLER}

As part of the fetal response to hypoxia (mainly due to placental insufficiency) selective redistribution of blood flow is incorporated to maintain perfusion of vital organs (brain, myocardium, adrenal glands). Assessment of MCA Doppler waveform will detect reduced resistance to flow with redistribution.

Arduni et $\mathrm{al}^{9}$ described a curvilinear relationship between the cerebral vascular response and hypoxia. Maximum vasodilation (to compensate for hypoxia) in the MCA is reached 2 weeks before the onset of fetal heart rate decelerations. Thus evidence of brain sparing is not in itself an indication for immediate delivery at very preterm gestation.

\section{DUCTUS VENOSUS DOPPLER}

Changes in Doppler velocimetry of the ductus venosus (DV) and the umbilical vein (UV) represent a late stage in cardiovascular decompensation. Urgent delivery is indicated with reversal of flow in DV or pulsation in UV.

In a high risk population an abnormal DV Doppler is a good predictor of birth acidemia with reported perinatal mortality rates of 63-100\% associated with absent and reversal of flow. It is superior to measurement of UA or MCA in predicting perinatal demise.

The combined assessment in fetal surveillance is considered best practice for all preterm intra uterine growth restricted fetuses:

- UA Doppler has the highest sensitivity and negative predictive value for poor perinatal outcome.

- DV and UV parameters have the best specificity and positive predictive values. ${ }^{10}$

\section{SEQUENCE IN DETERIORATION IN FETAL DOPPLER WAVEFORMS}

Initially there is a decrease in AFI and increase in UA Doppler, with positive end diastolic flow. This followed by decrease in resistance in MCA (brain sparing), and the presence of AEDF in the umbilical artery. Later the resistance in the DV increases together with REDF in the umbilical artery. The end 
point is reached when flow in the DV is reversed and becomes pulsatile in the UV.

\section{OTHER SURVEILLANCE TECHNIQUES BIOPHYSICAL SCORE OR PROFILE}

Some centres find the biophysical score useful because it provides multiple acute and chronic fetal physiologic parameters, is easy to perform and appears to be reliable. Fetal death within one week of a normal biophysical profile (BPP) is extremely rare and therefore this has a good negative predictive value. ${ }^{1}$

Performance of this investigation is however time consuming. According to a Cochrane review there is not enough evidence to evaluate the use of BPP as a test of fetal well-being in high risk pregnancies. ${ }^{11}$ The use of BPP in management of growth restriction is therefore not practised in our unit.

\section{AMNIOTIC FLUID VOLUME}

Amniotic fluid volume is an important component of longitudinal monitoring and gives an indication of renal perfusion of the fetus. It must be assessed serially by either measuring the single deepest vertical pocket or by calculating the amniotic fluid index (AFI).

Observational studies found that IUGR complicated by olighydramnios (defined as a single vertical pocket of fluid measuring less than $2 \mathrm{~cm} \times 1 \mathrm{~cm}$ or an AFI of less than 6 ) is associated with a sharply increased risk of perinatal mortality. Normal fluid volume is less frequently associated with growth restriction or fetal demise, unless congenital malformations or aneuploidy are present. ${ }^{1}$ Further analysis concluded that an AFI of/less than 5 is associated with a significant increased risk of caesarean section delivery for the indication of fetal distress and a low Apgar score at 5 minutes.

\section{CARDIOTOCOGRAPHY(CTG)}

Non-stress CTG is an indirect assessment of fetal central neurological status as reflected in its autonomic control of the fetal heart rate.

It remains the most common test used, despite paucity of evidence that it is associated with better neonatal outcome. Although a normal CTG has a good negative predictive value, in a systematic review the effectiveness could not be evaluated because of too little available evidence. ${ }^{12}$

Computerised CTG systems show better accuracy than the opinion of clinicians in predicting umbilical acidosis and low Apgar score. ${ }^{13}$ One small study looked at short term variability of $4,5 \mathrm{~mm}$ for predicting acidemia $(\mathrm{pH}<7)$ and showed a sensitivity of $100 \%$, specificity of $70 \%$, positive predictive value of $33 \%$ and negative predictive value of $100 \% \cdot{ }^{14}$

\section{FETAL BLOOD SAMPLING}

Intra uterine blood sampling sampling for evaluation of the fetal condition is not recommended. Adequate management is possible without invasive techniques.

\section{THE USE OF ANTENATAL CORTICOSTEROIDS}

Despite the findings from a large study that questioned the efficacy of cortciosteroids in preterm IUGR fetuses due to the inability to meet the transient increase in metabolic demand induced by steroids, the use of corticosteroids in IUGR is still recommended. ${ }^{15}$

According to the RCOG it is appropriate to administer a course of antenatal steroids to decrease neonatal pulmonary and central nervous system morbidity if preterm delivery occurs. It is known from multiple series that both spontaneous and indicated preterm deliveries are more common in IUGR fetuses.

The practise of administering multiple weekly courses of steroids gained support after publication of the Australasian Collaborate Trial of Repeat Steroids which showed a decrease in respiratory distress of $33 \mathrm{vs} .44 \%$, and the incidence of severe lung disease of 12 , compared to $20 \%$. However, longterm infant outcome results remain to be assessed. ${ }^{16}$

\section{TIMING OF DELIVERY}

There is still little consensus about the optimal timing of delivery. Delivery is indicated as soon as the risk for fetal death exceeds the risk of neonatal death. The decision is determined by both gestational age and fetal condition. The availability of neonatal care facilities and expert neonatal care providers must also form part of this equation.

The difficulties surrounding this decision were explored in the Growth Restriction Intervention Trial (GRIT). The authors recommended delayed delivery in very preterm gestations if there was uncertainty about the need for intervention. They found that deaths prior to hospital discharge were similar in both groups and that the immediate delivery group had fewer stillbirths but more neonatal and infant death..$^{17}$ During a 2 year follow up, the proportion of children with death or severe disability was similar for both group..$^{18}$

\section{REMOTE FROM TERM}

Normal umbilical artery doppler is reassuring with regard to the immediate fetal outcome. Therefore, prolongation of the pregnancy to gain further fetal maturity is reasonable. Absent end diastolic flow (AEDF) or reversed end diastolic flow (REDF) are ominous findings, and prompt delivery is often indicated because of high risk for fetal demise.

Changes in the venous circulation are generally later than those in the arterial circulation. They appear to be more predictive of impending adverse outcome and warrant immediate delivery regardless of gestational age. ${ }^{1}$

\section{TERM OR NEAR TERM}

Delivery can be delayed until 37 weeks when pulmonary maturity is more likely as long as antepartum fetal surveillance are reassuring and there is positive end diastolic flow present on Doppler evaluation. Pregnancies should not extend beyond 40 weeks

In the presence of maternal disease (e.g. hypertension), the finding of arrest of growth over a two to four week interval, and if the BPP score is low, and/or the UA Doppler velocimetry reveals absence or reversal of flow, delivery is indicated. However, every case must be individualized. ${ }^{1}$

\section{INTRAPARTUM MANAGEMENT}

IUGR fetuses may exist in a state of mild-to-moderate chronic oxygen and substrate deprivation. This may result in the occurrence of antepartum or intrapartum hypoxia and neonatal ischemic encephalopathy, fetal heart rate 
abnormalities, meconium aspiration, polycytemia,

hypoglycaemia, and other metabolic abnormalities. ${ }^{19}$

It is imperative to optimize timing of delivery and perform continuous intrapartum fetal monitorig and provide immediate skilled neonatal care.

\section{MODE OF DELIVERY}

Vaginal labour where the fetus has been found to have IUGR is still a reasonable option in the absence of any contraindications for normal vaginal delivery. As long as there is careful intrapartum monitoring that include continuous CTG and, where available, secondary tests (fetal scalp blood and ST segment analysis of the fetal electrocardiogram), and as long as rapid intervention when evidence of fetal intolerance appears, vaginal delivery can be attempted. The mode of delivery can be decided on based on the favourability of the cervix, the severity of IUGR, gestational age, fetal presentation and presence or absence of maternal complications. ${ }^{20}$

Some studies have shown the use of elective caesarean section for delivery of IUGR fetuses to be associated with lower rates of respiratory distress syndrome, neonatal seizures and deaths compared to vaginal delivery, but these did not reach statistical significance and the mothers were more likely to experience serious morbidity. ${ }^{21}$

\section{Conclusion}

IUGR remains a challenge for all obstetric health care providers. In spite of improvement in surveillance with the refinement of Doppler studies, timely diagnosis and optimal management remains complex in the high as well as low risk populations. Serious attention to antenatal surveillance may lead to improved antenatal care, better decision making re the timing and the mode of delivery, and in the individual case may lead to an improved outcome.

\section{References}

1. Resnik R. Fetal growth restriction: Evaluation and management. UptoDate Database. 2007.

http://www.ais.up.ac.za/health/eresoucesd.htm (accesed 28 February 2008).

2. Neilson JP. Symphysis-fundal height measurement in pregnancy. Cochrane Database Syst Rev 2000; 2:CD000944.

3. Chang TC, Robson SC, Boys RJ et al. Prediction of the small for gestational age infant: which ultrasonic measurement is best? Obstet Gynecol 1992;80(6):1030-1038.

4. Royal College of Obstetricians and Gynaecologists. The Investigation and Management of the Small-for-Gestational-Age Fetus. RCOG Guideline No 31.2002.

5. Hui, L. Diagnosis and management of fetal growth restriction: the role of fetal therapy. Best Pracice of Research clinical Obst and
Gynaecology. 2008;22:139-158.

6. Mendez, H. Introduction to the study of pre- and postnatal growth in humans: a review. Am J Genet 1985;20:63-67.

7. Karsdorp VH, van Vugt JM, van Geijn HP, et al. Clinical significance of absent or reversed end diastolic velocity waveforms in umbilical artery. Lancet. 1994;344:1664-8.

8. Alfirevic, Z, Neilson, JP. Doppler utrasonography in high risk pregnancies. Systematic review with meta analysis. Am J Obstet Gynecol 1995;172:1379-85.

9. Arduni, D, Rizzo $G$,Romanini C. Changes of pulsatility index from fetal vessels preceding the onset of late decelerations in growthretarded fetuses. Obstet Gynecol 1992;79(4): 605-610.

10. Baschat AA, Gembruch U,Weiner CP et al. Qualitative venous Doppler waveform analysis improves prediction of critical perinatal outcomes in premature growth-restricted fetuses. Ultrasound Obstet Gynecol 2003;22(3): 240-245.

11. Alfirevic Z, Neilson JP. Biophysical profile for fetal assessment in high risk pregnancies. Cochrane Database Syst Rev 2000; 2: CD000038.

12. Pattison $N \& M c C o w a n ~ L$. Cardiotocography for antepartum fetal assessment. Cochrane Database Syst Rev 2000; 2: CD001068.

13. Nielsen PV, Stigsby B, Nickelsen C et al. Computer assessment of the intrapartum cardiotocogram II. The value of compared with visual assessment. Acta Obstet Gynecol Scand 1988; 67(5): 461-464.

14. Anceschi MM, Ruozi-Berretta A, Piazze JJ et al. Computerized cardiotocography in the management of intrauterine growth restriction associated with Doppler velocimetry alterations. Int J Gynaecol Obstet2004; 86(3): 365-370.

15. Schaap, AH, Wolf,H, Bruinse, HW. Smolders-De Haas, H. Effects of antenatal corticosteroid administration on mortality and long-term morbidity in early preterm, growth-restricted infants. Obstet Gynecol.2001;97:954-9.

16. Crowther CA, Haslam RR, Hiller JE et al. Neonatal respiratory distress syndrome after repeat exposure to antenatal corticosteroids: a randomised controlled trial. Lancet 2006;367(9526):1913-1919.

17. The GRIT Study Group. A randomised trial of timed delivery for the compromised preterm fetus: short term outcomes and Bayesian interpretation. BJOG. 2003; 1 10:27-32.

18. The GRIT Study group. Infant wellbeing at 2 years of age in the Growth Restriction Intervention Trial (GRIT): multicentred randomised controlled trial. Lancet;364:513-9.

19. Low, JA, Boston, RW, Pancham, SR. Fetal asphyxia during the intrapartum period in intrauterine growth-retarded infants. Am J Obstet Gynecol.1972;113:351.

20. Maulik, D. Management of Fetal Growth Restriction: An EvidenceBased Approach. Clinical Obstetrics and Gynaecology.2006;49:32034.

21. Lee, HC, Gould, JB. Survival rates and mode of delivery for vertex preterm neonates according to small- or appropriate-forgestational-age status. Pediatrics 2006; 118(6): el 836-el844. 